\title{
Correlation between crack length and load drop for low-cycle fatigue crack growth in Ti-6242
}

\author{
R. Brommesson, M. Ekh, M. Hörnqvist
}

\begin{abstract}
For low-cycle fatigue tests with smooth bars the number of cycles to initiation is commonly defined from a measured relative drop in maximum load. This criterion cannot be directly related to the crack length, which is the actual measure of interest. In order to establish a relation between load drop and crack length for the high strength titanium alloy Ti-6242, this investigation compares data from controlled low-cycle fatigue crack growth tests and numerical simulations of these tests. To achieve sufficient accuracy in this relation, focus is given to modelling of mean stress relaxation. Three constitutive models, the Chaboche, the Ohno-Wang and the Chaboche with threshold, are evaluated with respect to experiments. Furthermore, a straightforward method with cycle-scaling of the material parameters are used to efficiently reduce calculation cost. It is shown that it is possible to determine the relationship between load drop and crack length from numerical simulations, provided that care is taken to relevant aspects of the materials stress-strain response. These results are also used to numerically evaluate the effect on load drop of the extensometer position relative to the crack.
\end{abstract}

\section{Introduction}

Strain controlled low-cycle fatigue (LCF) tests are a common tool for establishing and calibrating crack initiation criteria for fatigue analysis in engineering structures and components. The number of cycles to initiation $\left(N_{i}\right)$ is usually defined as the point where a given measure exceeds some pre-determined limit. In strain controlled testing of smooth specimens, the most common measure is the $X \%$ load drop criteria $[1,2]$, where initiation is defined as the point where the maximum load has dropped below $\mathrm{X} \%$ of the saturated stable value. In the case of continuously increasing or decreasing maximum stress the load drop compared to the extrapolated values of stress could be used [3, 4], as schematically shown in Fig. 1.

The $\mathrm{X} \%$ load drop criterion is straightforward to apply and it also has the practical advantage that the test can be stopped prior to fracture; thus, avoiding risks of extensometer damage and destruction of fracture surface features. However, the inherent drawback of the criterion is that it is not explicitly related to the size of the crack, which is the measure that is of actual interest. For example, if only LCF data is to be used to assess the life of a component, the crack size at the point of initiation must be known and compared to the dimensions of the critical location in the component. Moreover, to allow subsequent crack propagation analysis the crack size at the point of initiation must be known. It is therefore of interest to investigate how the drop in maximum load and crack length are related during LCF testing. Furthermore, for round test bars, where the crack initiation may occur on 


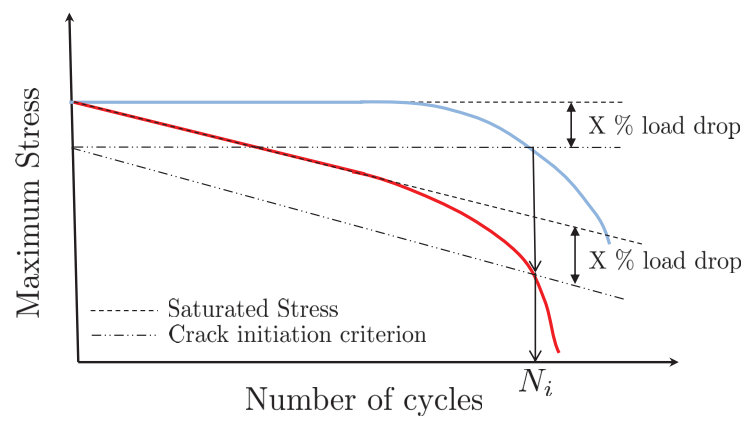

Figure 1: Typical application of the X \% load drop criteria to tests with constant or continuously decreasing maximum stress.

random location along the length and circumference of the gauge section, the location of the extensometer relative to the point of initiation can play a dominating part in the relation between crack length and load drop [5]. This fact is generally not regarded in LCF testing practice, but could be accounted for in numerical simulations. Additionally, if a relation between load drop and crack length was established in numerical simulations, this could be used to estimate crack length from already performed LCF tests, provided that the crack initiation sites were marked in the testing procedure. By gaining more information from available test data the need for further tests could potentially be reduced, which would result in economical benefits.

The relation between load drop and crack length for high strain fatigue crack growth tests of Ti-6242 was investigated in [6]. The investigation showed that a relation between load drop and crack length could be established in numerical finite element (FE) examples, provided that the mean stress relaxation observed for the tests was properly taken care of. In the investigation the slow mean stress relaxation was accounted for by compensating the experimental data. However, it was noted that this procedure is only possible if experiments of sufficient quality are available. A more general approach is to let the mean stress relaxation be included in the numerical simulations. This approach requires that constitutive models are applied that are able to predict mean stress relaxation. Mean stress relaxation and ratchetting are closely linked material processes, obtained for strain controlled and stress controlled loading, respectively. To model these processes many constitutive models have been proposed with different levels of accuracy and complexity, see e.g. overviews in [7, 8]. In addition, to account for the mean stress relaxation the numerical simulations must also be run for the number of loading cycles used in the experiments.

Modelling of many load cycles can result in that the computational cost of the numeric simulations quickly raises above reasonable limits and more time efficient integration methods are needed. For example, efficient time integration technique with extrapolation of load cycles like the one used in $[9,10]$. The drawback of such methods is the requirement of extensive implementation in the FE-code, which can be difficult to achieve in a commercial code. Another example of efficient integration is to express the constitutive equations in the cycle domain $[11,12]$. However, this method requires a reformulation of the constitutive equations, which traditionally are expressed in the time domain. 
In this work, the high strain fatigue crack propagation tests used in [6] are revisited. FEmodels of the test specimens are used to establish the relation between load drop and crack length. In order to accurately predict the load drop the mean stress relaxation observed in the tests is modelled. Three constitutive models are evaluated, the Chaboche model [13, 14] used in the previous investigation [6]; the Ohno-Wang model [15]; and the Chaboche with threshold model $[16,17,18]$. The latter two models are better suited for predicting the mean stress behaviour of tests with different strain ratios, cf. [8]. To account for the high computational cost, for the many loading cycles needed in the simulations, a straightforward approach using cycle-scaling of the calibrated material parameters is applied. Finally, the effect on load drop based on the extensometer position with respect to the crack is evaluated numerically in FE-simulations.

\section{Experiment}

\subsection{Fatigue crack growth testing}

The material selected for this investigation is a high temperature $\alpha / \beta$ Ti-6Al-2Sn-4Zr$2 \mathrm{Mo}(-0.1 \mathrm{Si})$ alloy (Ti-6242), with the composition given in weight percent. The alloy is commonly applied in aero engine compressors. Surface flawed fatigue crack growth specimens of Kb-type [19] (see Fig. 2) were machined from bar material with the tensile axis in the longitudinal direction. The specimens had a rectangular cross-section of $10.2 \times 4.3 \mathrm{~mm}$ in the $32 \mathrm{~mm}$ long gage section. A $0.2 \mathrm{~mm}$ deep starter notch, centered on the wide face of the specimen, was machined using electric discharge machining (EDM). The specimens were instrumented for direct current potential drop (DCPD) measurements by spot welding PD probes across the crack and at a location away from the cracked cross-section to provide a reference signal. Fatigue cracks of length $0.6-0.7 \mathrm{~mm}$ were generated in stress control at room temperature using a $10 \mathrm{~Hz}$ trapezoidal waveform with $\mathrm{R}_{\sigma}=\sigma_{\min } / \sigma_{\max }=0$, at linear elastic fracture mechanics (LEFM) conditions.

High strain fatigue crack growth tests were carried out in a servo-hydraulic testing machine at $350{ }^{\circ} \mathrm{C}$, heated using a conventional resistance furnace and controlled by a thermo-couple attached to the specimen. Total strain control was employed with the strain measured by a $12 \mathrm{~mm}$ gage length extensometer attached to the side face of the specimen, centered around the cracked cross-section. The total applied strain range was $1.2 \%$ at a frequency of $0.5 \mathrm{~Hz}$ for all tests, and strain ratios $R_{\epsilon}=\epsilon_{\min } / \epsilon_{\max }=0$ and 0.6 were used with duplicate tests for each condition. During the tests, DCPD signal was continuously recorded and translated to crack length through an experimentally obtained calibration curve. The recorded signal was normalized by the reference signal in order to avoid effects of fluctuating temperatures or currents during the test. Maximum and minimum stresses were recorded for every 10 cycles, and full hysteresis loops were recorded at logarithmic intervals.

\subsection{Fatigue crack growth testing - Results}

The global stress-strain response, in terms of strain measured by the extensometer and nominal stress based on the un-cracked area, for one specimen for each $\mathrm{R}_{\epsilon}$-ratio, is shown in Fig. 3a. From the figure it is clear that the specimens undergo extensive plasticity during the first loading excursion. Furthermore, the specimens also undergo global cyclic plasticity throughout the tests. 

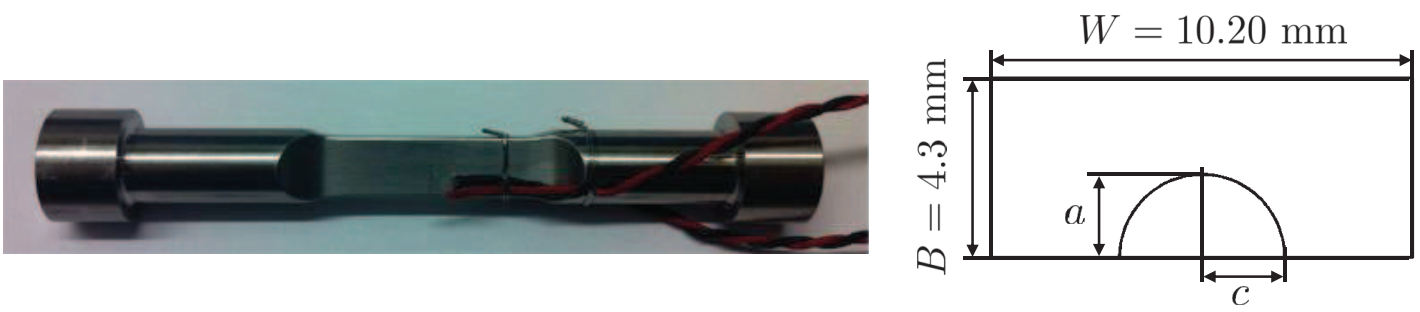

Figure 2: A Kb-type specimen with attached potential drop probes, and geometry of the specimen cross-section and crack.

The material exhibits a slight cyclic hardening, as seen from the increasing stress amplitude development in Fig. 3c. The apparent softening observed after about 1000 cycles in Fig. 3b and Fig. 3c is related to the loss of cross-sectional area from the increase in crack length rather than a material softening. From Fig. 3c it can be observed that the mean stress decreases with number of cycles, but does not reach zero during the duration of the test. This material behaviour has an effect on the measured load drop and, therefore, it is important when modelling the relation between load drop and crack length to give an accurate description of the mean stress relaxation.

In Fig. 3d the relative load drop is given as a function of the relative reduction in crosssection area. The relative load drop, $\sigma_{\text {drop }}$, is measured as the reduction in maximum stress relative to the maximum stress at the 500 th loading cycle, $\sigma_{\max , 500}$, according to

$$
\sigma_{\text {drop }}(n)=1-\frac{\sigma_{\max }(n)}{\sigma_{\max , 500}}
$$

where $n$ is the cycle number. The 500th cycle is chosen as reference for this measure since, at this cycle, all four tests have reached a semi-stable stress-strain response and the influence of the crack is still small. The relative reduction in cross-section area, $A_{\text {red }}$, is calculated as

$$
A_{\text {red }}(n)=\frac{A_{\text {Crack }}(n)}{A_{0}}
$$

where $A_{0}$ is the area of the undamaged cross-section and $A_{\text {Crack }}$ is the area of the crack, which is known from experiments. From Fig. 3d it can be noted that the relationship between relative load drop and relative reduction in cross-section is independent of the strain ratio, $\mathrm{R}_{\epsilon}$.

\section{Modelling}

\subsection{Constitutive models for prediction of mean stress relaxation}

The mean stress relaxation observed for the high strain tests in Section 2.2 is an important factor contributing to the load drop, as discussed in [6]. Constitutive modelling of mean stress relaxation (in strain controlled loading) and ratchetting (in stress controlled loading) has been a subject for many researchers over the years cf. e.g. [7]. The key for modelling mean stress 


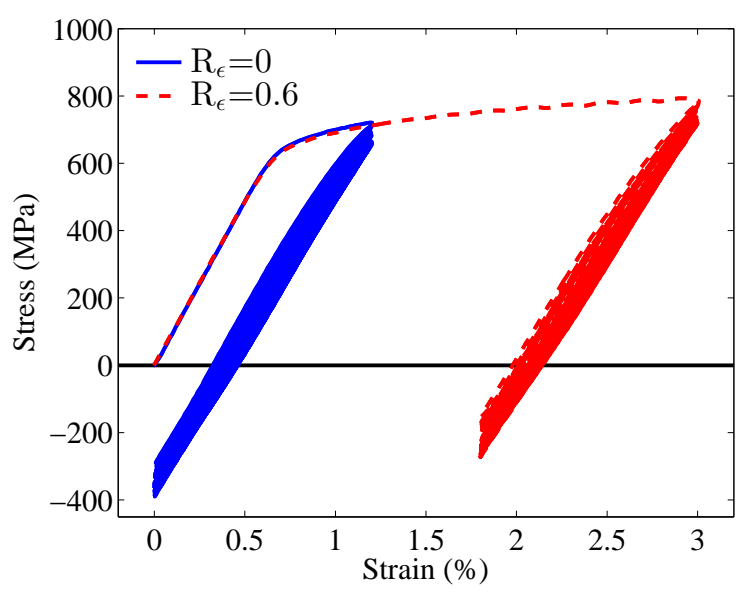

(a)

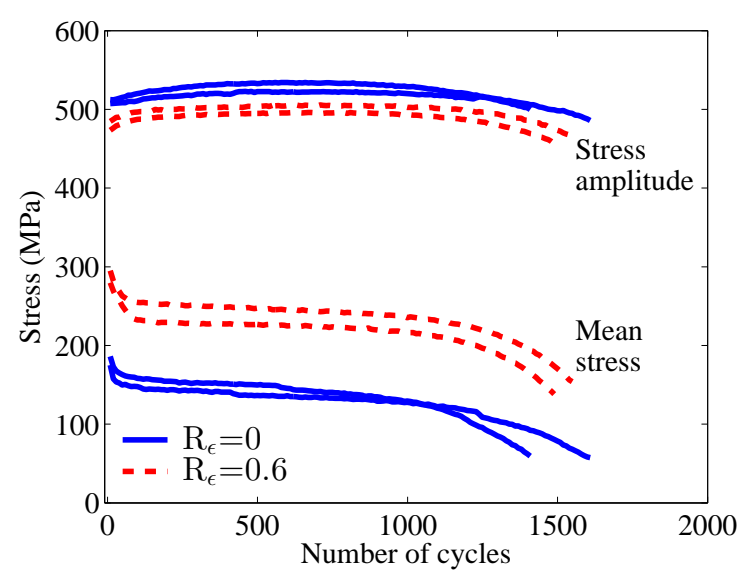

(c)

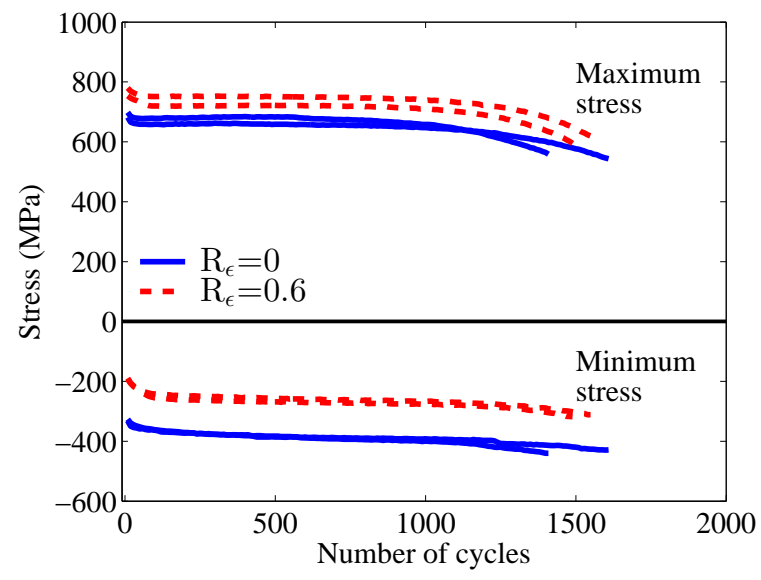

(b)

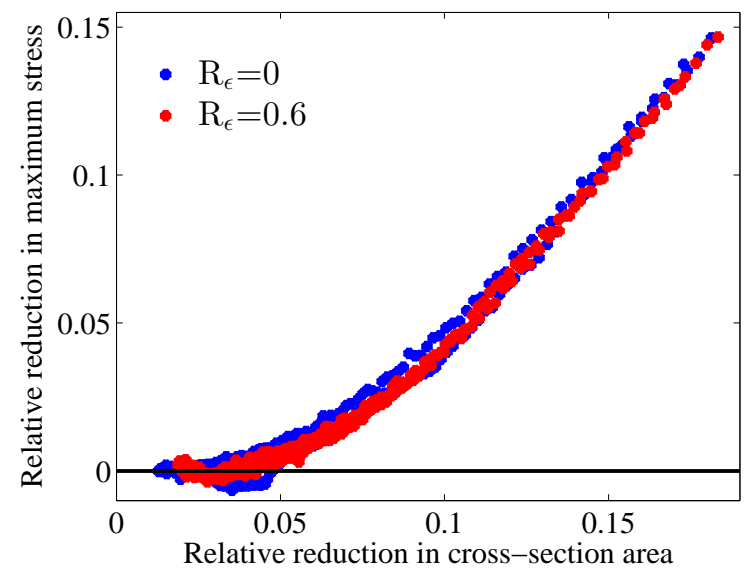

(d)

Figure 3: Results from the high strain fatigue crack growth tests. (a) shows the stress-strain response of two tests with $\mathrm{R}_{\epsilon}=0$ and $\mathrm{R}_{\epsilon}=0.6$. The cyclic behaviour of all four tests are shown in (b) as the development of maximum and minimum stress and in (c) as the development of stress amplitude and mean stress with cycle number. In (d) the relative load drop is given as a function of the relative reduction in cross-section area. 
relaxation and ratchetting is to define proper evolution of backstress, i.e. the description of kinematic hardening.

In this investigation three material models are evaluated with respect to the mean stress relaxation observed in the experiments. The three models are the Chaboche, the Ohno-Wang and the Chaboche with threshold. The Chaboche model has been selected because it is a fairly simple model commonly implemented in commercial software, e.g. Abaqus [20]. The Ohno-Wang model and the Chaboche with threshold model have been included in this work due to that they are often discussed and used when improved predictions of mean stress relaxation or ratchetting behaviour are needed, see e.g. [8, 18, 21, 22].

The three models use the same modelling framework and are only differentiated by the description of the kinematic hardening. In the modelling framework a split of the stress tensor, $\boldsymbol{\sigma}$, into a volumetric, $\sigma_{\mathrm{vol}}$, and a deviatoric, $\boldsymbol{\sigma}_{\mathrm{dev}}$, part is used

$$
\boldsymbol{\sigma}=\boldsymbol{\sigma}_{\mathrm{dev}}+\frac{1}{3} \sigma_{\mathrm{vol}} \boldsymbol{I}
$$

where $\boldsymbol{I}$ is the 2 nd order identity tensor. Moreover, $\boldsymbol{\sigma}_{\mathrm{dev}}$ and $\sigma_{\mathrm{vol}}$ are, by an assumption of isotropic linear elasticity, related to the elastic volumetric strain, $\epsilon_{\mathrm{e}, \mathrm{vol}}$, and elastic deviatoric strain, $\boldsymbol{\epsilon}_{\mathrm{e}, \mathrm{dev}}$, according to

$$
\begin{aligned}
& \boldsymbol{\sigma}_{\mathrm{dev}}=2 \mathrm{G} \boldsymbol{\epsilon}_{\mathrm{e}, \mathrm{dev}} \\
& \sigma_{\mathrm{vol}}=3 \mathrm{~K}_{\mathrm{b}} \epsilon_{\mathrm{e}, \mathrm{vol}}
\end{aligned}
$$

where $\mathrm{G}$ and $\mathrm{K}_{\mathrm{b}}$ are the shear and bulk modulus, respectively. The total strain, $\boldsymbol{\epsilon}$, is additively decomposed into its elastic and plastic parts

$$
\epsilon=\epsilon_{\mathrm{e}}+\epsilon_{\mathrm{p}}
$$

The elastic and plastic material behaviour is separated by a von Mises yield function defined as

$$
\Phi=\sqrt{\frac{3}{2}}\left|\boldsymbol{\sigma}_{\mathrm{dev}}-\boldsymbol{B}\right|-\sigma_{\mathrm{y}}
$$

where $\sigma_{\mathrm{y}}$ is the yield stress and $\boldsymbol{B}$ is the total backstress representing the kinematic hardening. Furthermore, associative evolution is assumed for the plastic strain

$$
\dot{\boldsymbol{\epsilon}}_{\mathrm{p}}=\dot{\lambda} \frac{\partial \Phi}{\partial \boldsymbol{\sigma}}=\dot{\lambda} \sqrt{\frac{3}{2}} \frac{\boldsymbol{\sigma}_{\mathrm{dev}}-\boldsymbol{B}}{\left|\boldsymbol{\sigma}_{\mathrm{dev}}-\boldsymbol{B}\right|}
$$

where the plastic multiplier is determined from the loading/unloading conditions

$$
\Phi \dot{\lambda}=0, \quad \dot{\lambda} \geq 0, \quad \Phi \leq 0
$$

It can be noted that, the small amount of cyclic hardening observed in Fig. 3 has not been accounted for in the material model, since its effect on the load drop is small. If the cyclic hardening is to be accounted for this could easily be included in the material models in the form of e.g. isotropic hardening [8]. 
The evolution of the backstress is controlling the mean stress relaxation behaviour. In the Chaboche model [14] multiple nonlinear kinematic hardening rules of Armstrong-Frederick type [13] are used, according to

$$
\dot{\boldsymbol{B}}_{i}=\frac{2}{3} C_{i} \dot{\boldsymbol{\epsilon}}_{\mathrm{p}}-\gamma_{i} \boldsymbol{B}_{i} \dot{\lambda}
$$

where $C_{i}$ is the hardening modulus and the saturation value, $B_{\infty, i}$, for the hardening can be introduced as $B_{\infty, i}=C_{i} / \gamma_{i}$. The total backstress, $\boldsymbol{B}$, is obtained from the summation of the backstresses

$$
\boldsymbol{B}=\sum_{i=1}^{\mathrm{n}_{\mathrm{B}}} \boldsymbol{B}_{i}
$$

where $\mathrm{n}_{\mathrm{B}}$ is the number of kinematic hardening terms included in the model.

It is well known that the nonlinear formulation of the kinematic hardening can result in overestimates of the mean stress relaxation or ratchetting behaviour $[7,8]$. By including more than one backstress in the model and letting one of them be linear or close to linear, i.e. $\gamma_{i} \cong 0$, a decrease in the rate of mean stress relaxation or ratchetting can be obtained. However, this approach requires an overly stiff kinematic hardening effect. Therefore, it is impossible to obtain reasonable predictions of both cyclic and monotonic behaviour, using this model and a single set of material parameters.

To improve predictions of mean stress relaxation and ratchetting behaviour without compromising the initial and monotonic behaviour of the model, a threshold effect was included in the kinematic hardening [17], according to

$$
\dot{\boldsymbol{B}}_{i}=\frac{2}{3} C_{i} \dot{\boldsymbol{\epsilon}}_{\mathrm{p}}-C_{i}\left\langle\frac{\sqrt{\frac{3}{2}}\left|\boldsymbol{B}_{i}\right|-w_{i} B_{\infty_{i}}}{\left[1-w_{i}\right] B_{\infty_{i}}}\right\rangle^{m_{i}} \frac{\boldsymbol{B}_{i}}{\sqrt{\frac{3}{2}}\left|\boldsymbol{B}_{i}\right|} \dot{\lambda}
$$

The threshold effect is obtained by the Macaulay-brackets $<\bullet>$, which for $\sqrt{3 / 2}\left|\boldsymbol{B}_{i}\right| \leq$ $w_{i} B_{\infty_{i}}$ will give a zero value and hence a linear kinematic term, whereas for $\sqrt{3 / 2}\left|\boldsymbol{B}_{i}\right|>$ $w_{i} B_{\infty_{i}}$ a nonlinear kinematic term will be the result. Therefore the parameter $w_{i}$ will have a large impact on the mean stress relaxation, whereas the exponent $m_{i}$ adds to the degrees of freedom of the model. It can be noted that for a choice of parameter $w_{i}=0$ and $m_{i}=1 \mathrm{Eq}$. 12 reduces to the Armstrong-Frederick rule in Eq. 10.

The last material model evaluated is the Ohno-Wang model [15]. The model is built on an idea of introducing a critical state of dynamic recovery,

$$
\dot{\boldsymbol{B}}_{i}=\frac{2}{3} C_{i} \dot{\boldsymbol{\epsilon}}_{\mathrm{p}}-\gamma_{i} \mathcal{H}\left(\frac{3}{2} \boldsymbol{B}_{i}: \boldsymbol{B}_{i}-\left[\frac{C_{i}}{\gamma_{i}}\right]^{2}\right)\left\langle\dot{\boldsymbol{\epsilon}}_{\mathrm{p}}: \frac{\boldsymbol{B}_{i}}{\sqrt{\frac{3}{2}}\left|\boldsymbol{B}_{i}\right|}\right\rangle \boldsymbol{B}_{i}
$$

where $\mathcal{H}(\bullet)$ is the Heaviside function. By this formulation a linear hardening is obtained until the critical state, $C_{i} / \gamma_{i}$, is reached, whereafter the hardening rule cease to evolve. This formulation leads to a multilinear model, which does not produce any uniaxial mean stress relaxation or ratchetting. However, by a slight alteration of the model a nonlinearity can be 
included in the transition to the critical state

$$
\dot{\boldsymbol{B}}_{i}=\frac{2}{3} C_{i} \dot{\boldsymbol{\epsilon}}_{\mathrm{p}}-\gamma_{i}\left[\frac{\sqrt{\frac{3}{2}}\left|\boldsymbol{B}_{i}\right|}{C_{i} / \gamma_{i}}\right]^{m_{i}}\left\langle\dot{\boldsymbol{\epsilon}}_{\mathrm{p}}: \frac{\boldsymbol{B}_{i}}{\sqrt{\frac{3}{2}}\left|\boldsymbol{B}_{i}\right|}\right\rangle \boldsymbol{B}_{i}
$$

In this formulation, which is the one used in this paper, the Heaviside function has been replaced by a multiplier with power $m_{i}$. The size of $m_{i}$ controls the rate of mean stress relaxation. Decreasing the value of $m_{i}$ will increase the rate of mean stress relaxation whereas for high values a multilinear response is, yet again, obtained.

The three selected constitutive models are calibrated in an in-house code according to a procedure described in [23]. For calibration of the cyclic stress-strain behaviour the influence of cracks should generally be avoided [24]. In this investigation, the first 800 loading cycles of the high strain crack propagation tests are used. For these cycles, cracks are present in the test specimens, but the influence of the cracks on the material behaviour is considered to be small. Additionally, it can be mentioned that 3 backstresses were used in the calibration of the Chaboche model, whereas 2 backstresses were considered to give sufficiently accurate responses for the Ohno-Wang model and the Chaboche with threshold model.

In Fig. 4 results from the calibrated material models are shown. From Fig. 4b and Fig. 4c, it can be seen that all three models predict the cyclic hysteresis loop with good accuracy. All three models are also able to predict the mean stress relaxation observed for the first 800 cycles in Fig. 4d. However, it should be noted that for the Ohno-Wang and the Chaboche with threshold models only one set of material parameters are required. Whereas, for the Chaboche model, two separate sets of material parameters are used for the two loading conditions, $\mathrm{R}_{\epsilon}=0$ and $\mathrm{R}_{\epsilon}=0.6$. In order to achieve a reasonable accuracy of the cycle predictions an overly stiff kinematic hardening effect is needed for this model. As a result, a poor fit with respect to experiments is obtained for the first cycle, where the model response overestimates the maximum stress. This drawback of the Chaboche model can be seen in the first loading cycle in Fig. 4a, where results for both loading conditions are displayed. Furthermore, it can be noted that very similar results were obtained for the Chaboche with threshold model and the Ohno-Wang model. The fact that similar responses can be obtained with the two models was also pointed out in [8].

\subsection{Cycle-scaling of material parameters}

The mean stress relaxation observed in the experiments is a slow process. To correctly predict it in an FE-analysis all cycles should be simulated, which is computational expensive. To reduce calculation costs efficient integration techniques are needed, cf. e.g. [9, 11, 12].

In this work we propose a straightforward method consisting of cycle-scaling of experiments and material parameters. The cycles of the experiments used for the calibration of the material parameters are scaled 1:1, 1:10 and 1:100. As an example, cycle 1000 of the experiment (1:1 scale) is represented by cycle 100 and 10 in the scaled versions (1:10 and 1:100) of the experiments. The material parameters are re-calibrated based on the scaled experiments. (It can be noted that the first loading cycle of the experiments is denoted as cycle 0 and treated equally for all three cycle-scales.) To limit the effort in the re-calibration procedure and increase the applicability of the method, as many of the material parameters 


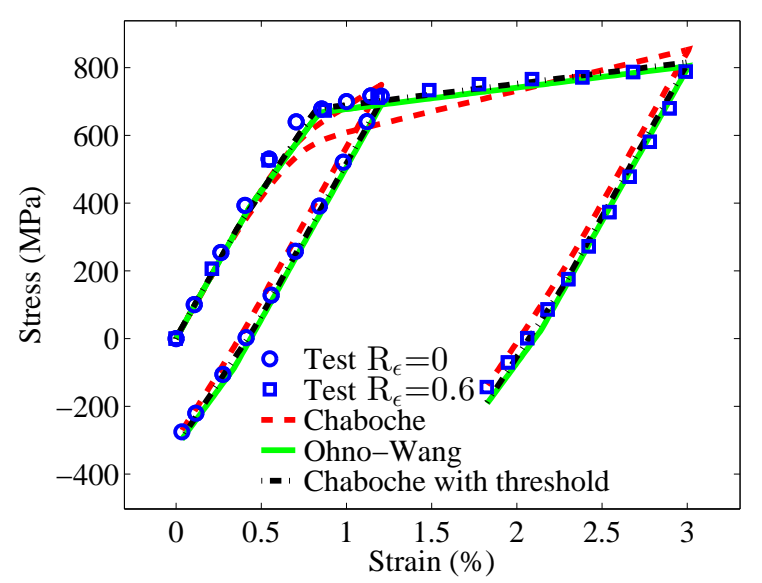

(a)

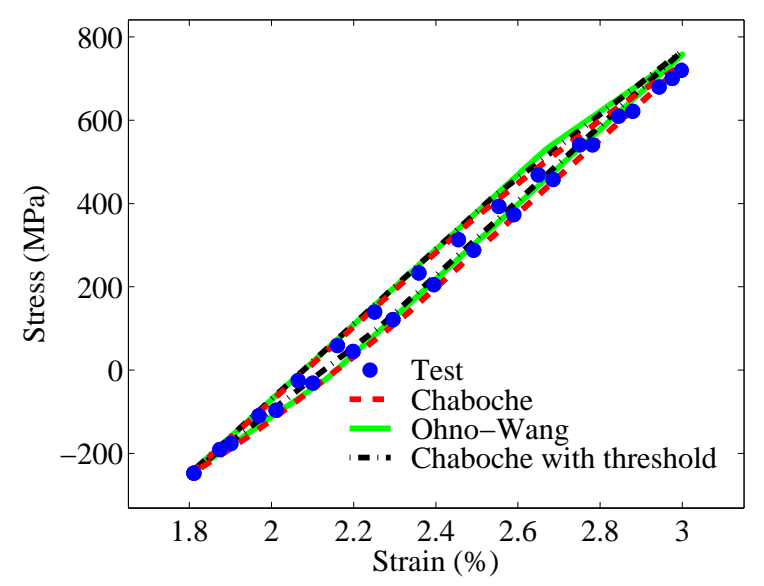

(c)

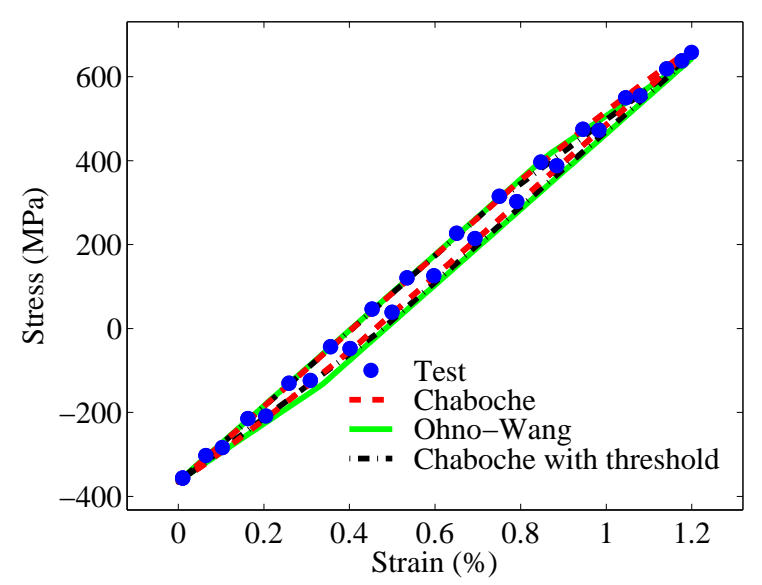

(b)

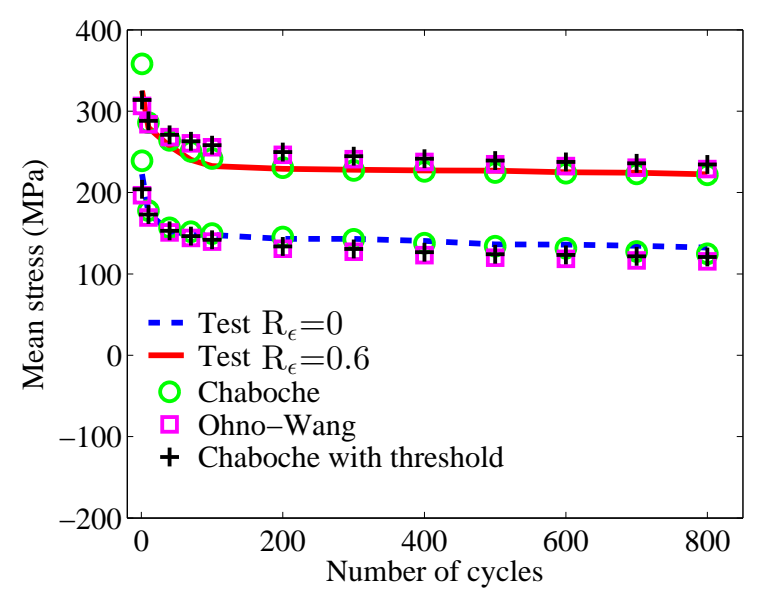

(d)

Figure 4: Calibrated model response for the three material models ( the Chaboche, OhnoWang and Chaboche with threshold models), compared to results from experiments. (a) Results for the initial loading cycle for both loading conditions, $R_{\epsilon}=0$ and $R_{\epsilon}=0.6$, and results for cycle 100 for (b) $R_{\epsilon}=0$ and (c) $R_{\epsilon}=0.6$. (d) The cyclic development of mean stress with cycle number. 


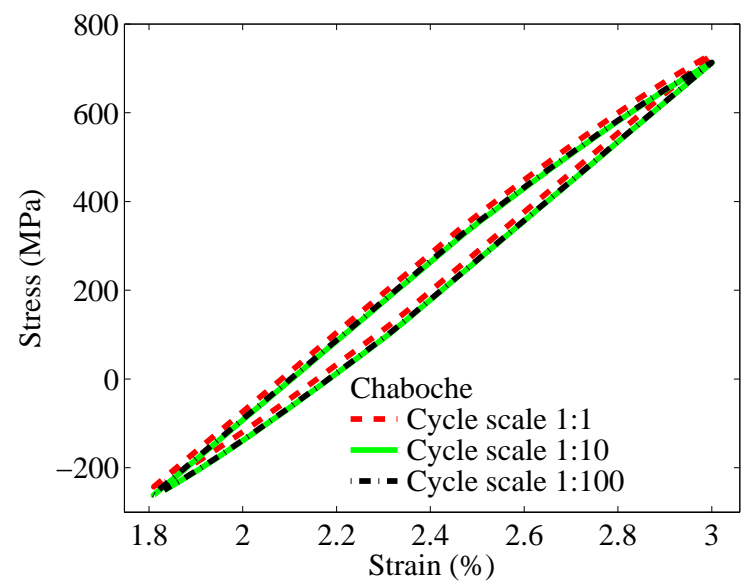

(a)

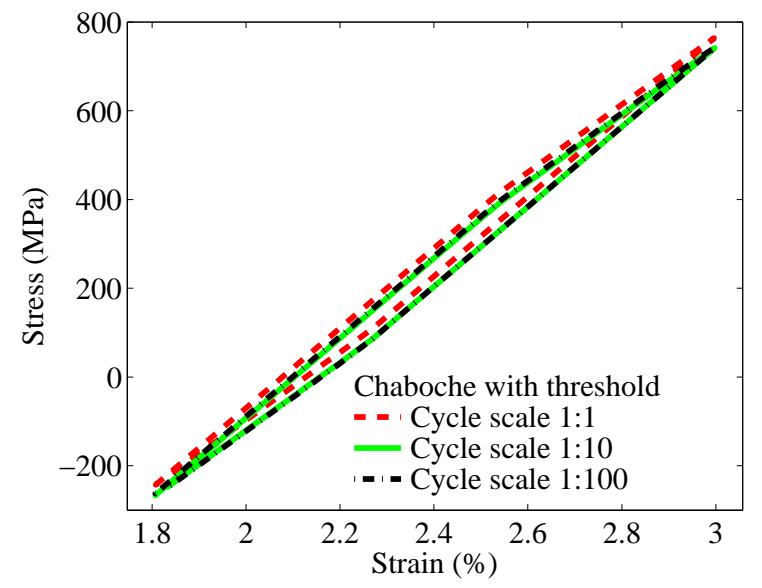

(b)

Figure 5: Calibrated model response for cycle 100 from all three cycle-scales and $R_{\epsilon}=0.6$ for a) the Chaboche model and b) the Chaboche with threshold model.

Table 1: Calibrated material parameters for the Chaboche model and $\mathrm{R}_{\epsilon}=0$.

\begin{tabular}{ccccccccc}
\hline Cycle-scale & $\mathrm{E}(\mathrm{GPa})$ & $\sigma_{y}(\mathrm{MPa})$ & $H_{1}(\mathrm{GPa})$ & $\gamma_{1}(-)$ & $H_{2}(\mathrm{GPa})$ & $\gamma_{2}(-)$ & $H_{3}(\mathrm{GPa})$ & $\gamma_{3}(-)$ \\
\hline $1: 1$ & 90 & 360 & 640 & 3050 & 14 & 54 & 36 & $3.4 \mathrm{e}^{-1}$ \\
$1: 10$ & 90 & 360 & 640 & 3050 & 14 & 54 & 28 & $2.8 \mathrm{e}^{-2}$ \\
$1: 100$ & 90 & 360 & 640 & 3050 & 14 & 54 & 19 & $6.7 \mathrm{e}^{-2}$ \\
\hline
\end{tabular}

as possible were kept from the original calibration (cycle-scale 1:1). It was observed that the scaled experiments could be well modelled by only changing the parameters controlling the mean stress relaxation. The parameters for the different cycle scales and models are summarised in Table 1-4.

In Fig. 5 the model response representing cycle 100, i.e. cycle 1, 10 and 100 for the scales of 1:100, 1:10 and 1:1, is shown for the Chaboche model and the Chaboche with threshold model. It can be observed that the differences in model response for this hysteresis loop are very small. Similar results were obtained for the Ohno-Wang model and the Chaboche with threshold model. In Fig. 6 the mean stress development for all models and cycle scales are shown. For the model responses, the number of cycles has been multiplied by its respective scale-factor. It can be noted that there are slight differences in the model results for the different scales. However, in general all model predictions correspond well to the experiments.

Table 2: Calibrated material parameters for the Chaboche model and $R_{\epsilon}=0.6$.

\begin{tabular}{ccccccccc}
\hline Cycle-scale & $\mathrm{E}(\mathrm{GPa})$ & $\sigma_{y}(\mathrm{MPa})$ & $H_{1}(\mathrm{GPa})$ & $\gamma_{1}(-)$ & $H_{2}(\mathrm{GPa})$ & $\gamma_{2}(-)$ & $H_{3}(\mathrm{GPa})$ & $\gamma_{3}(-)$ \\
\hline $1: 1$ & 90 & 300 & 480 & 1810 & 3.7 & 8.8 & 11 & $2.5 \mathrm{e}^{-2}$ \\
$1: 10$ & 90 & 300 & 480 & 1810 & 3.7 & 8.8 & 8.8 & $9.6 \mathrm{e}^{-4}$ \\
$1: 100$ & 90 & 300 & 480 & 1810 & 3.7 & 8.8 & 7.2 & $2.4 \mathrm{e}^{-3}$ \\
\hline
\end{tabular}




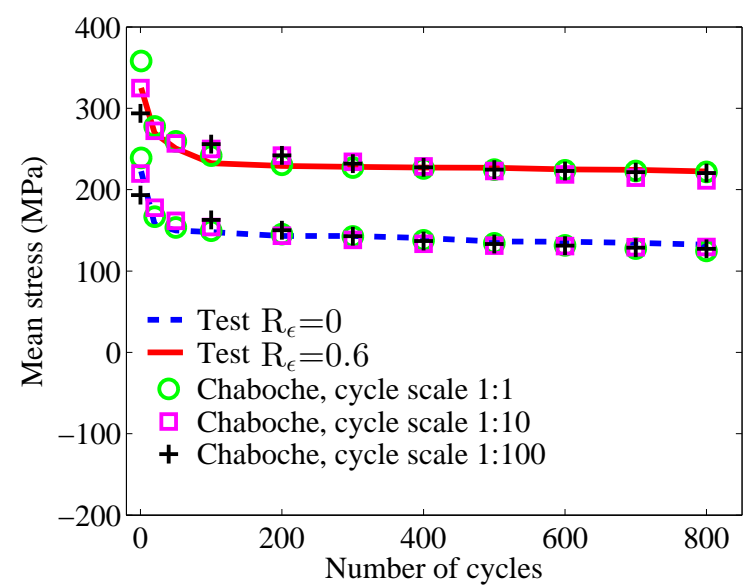

(a)

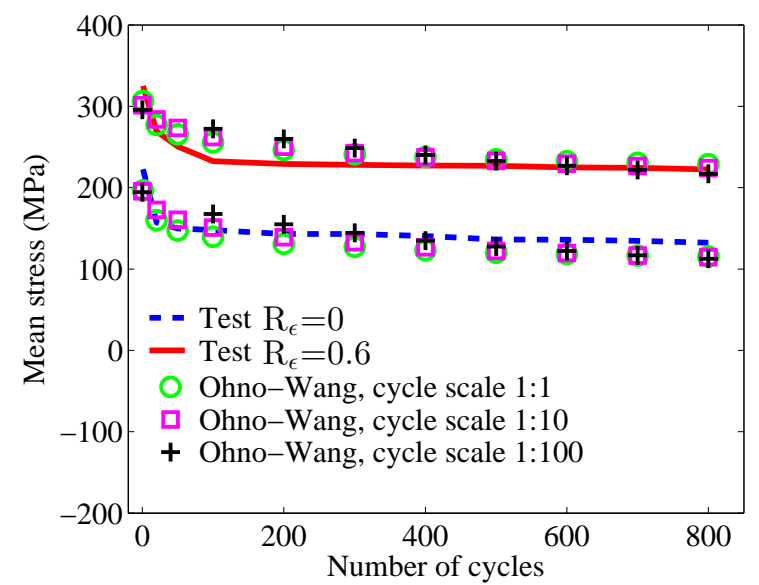

(b)

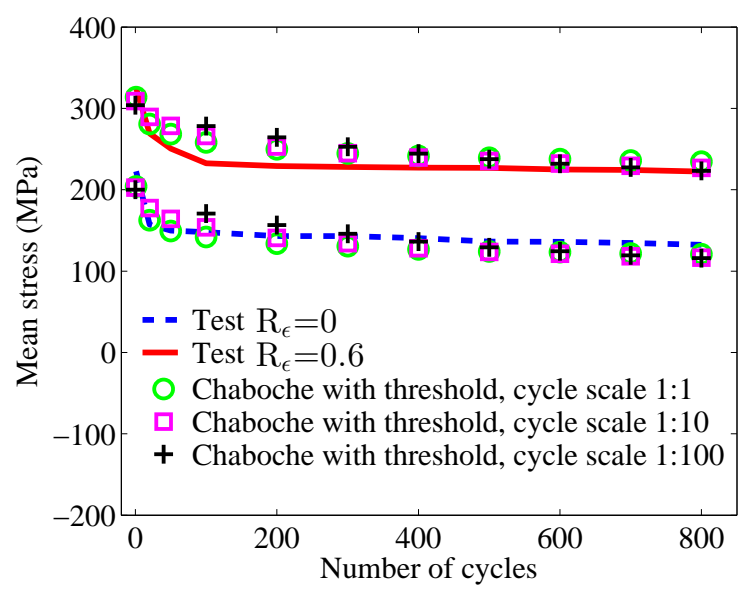

(c)

Figure 6: Calibrated cycle-scaled results for the development of mean stress with cycle number for the three material models, (a) the Chaboche model, (b) the Ohno-Wang model and (c) the Chaboche with threshold model.

Table 3: Calibrated material parameters for the Ohno-Wang model.

\begin{tabular}{ccccccccc}
\hline Cycle-scale & $\mathrm{E}(\mathrm{GPa})$ & $\sigma_{y}(\mathrm{MPa})$ & $C_{1}(\mathrm{GPa})$ & $\gamma_{1}(-)$ & $m_{1}(-)$ & $C_{2}(\mathrm{GPa})$ & $\gamma_{2}(-)$ & $m_{2}(-)$ \\
\hline $1: 1$ & 90 & 390 & 280 & 1030 & 15 & 6.8 & 0.0 & 1.0 \\
$1: 10$ & 90 & 390 & 280 & 1030 & 8.1 & 6.8 & 0.0 & 1.0 \\
$1: 100$ & 90 & 390 & 280 & 1030 & 2.3 & 6.7 & 0.0 & 1.0 \\
\hline
\end{tabular}

Table 4: Calibrated material parameters for the Chaboche with threshold model.

\begin{tabular}{ccccccccccc}
\hline Cycle-scale & $\mathrm{E}(\mathrm{GPa})$ & $\sigma_{y}(\mathrm{MPa})$ & $C_{1}(\mathrm{GPa})$ & $\gamma_{1}(-)$ & $w_{1}(-)$ & $m_{1}(-)$ & $C_{2}(\mathrm{GPa})$ & $\gamma_{2}(-)$ & $w_{2}(-)$ & $m_{2}(-)$ \\
\hline $1: 1$ & 90 & 330 & 500 & 1450 & $5.7 \mathrm{e}^{-1}$ & 7.0 & 6.8 & 0.0 & 0.0 & 1.0 \\
$1: 10$ & 90 & 330 & 500 & 1450 & $4.8 \mathrm{e}^{-1}$ & 5.0 & 6.6 & 0.0 & 0.0 & 1.0 \\
$1: 100$ & 90 & 330 & 500 & 1450 & $5.9 \mathrm{e}^{-1}$ & 1.0 & 6.4 & 0.0 & 0.0 & 1.0 \\
\hline
\end{tabular}


Table 5: Approximate number of cycles to obtain crack lengths of 1, 1.5 and $2 \mathrm{~mm}$ in the low-cycle fatigue crack growth tests.

\begin{tabular}{c|ccc}
\hline Crack length & $1 \mathrm{~mm}$ & $1.5 \mathrm{~mm}$ & $2 \mathrm{~mm}$ \\
$\mathrm{R}_{\epsilon}=0$, Number of cycles & 400 & 1000 & 1300 \\
$\mathrm{R}_{\epsilon}=0.6$, Number of cycles & 600 & 1100 & 1400 \\
\hline
\end{tabular}

\section{Results}

\subsection{FE-analysis}

To predict the relationship between load drop and crack length, observed for the high strain crack growth test, 3D FE-models of the test specimens were generated and analysed in the commercial code Abaqus [20]. Due to symmetry, only a quarter of the test specimen geometry was modelled, using C3D4 and C3D8 elements. At crack edges a finer mesh using only C3D8 elements was applied. Approximately 20000 elements were used for each model.

To simplify the analyses, no propagation of cracks was simulated. Instead, models for three different crack sizes $(a=c=1,1.5$ and $2 \mathrm{~mm})$ were used. The cracks were modelled by free nodes and a (friction free) contact analysis with a rigid surface was applied to prevent crack nodes from penetrating the symmetry crack plane during compression. In each case the number of simulated load cycles are equal to the number of cycles needed in the experiments to propagate the crack to the size used in the FE-model. In Table 5 the cycle numbers corresponding to the average number of cycles to reach the specific crack lengths in the two duplicate high strain fatigue tests are given.

It can be noted that the data presented in Table 5 indicate roughly $\mathrm{R}_{\epsilon}$ independent crack growth rates. However, this is not strictly true, since the stress range, $\Delta \sigma$, differs between the two test conditions. In the stabilized state, the stress amplitude at $\mathrm{R}_{\epsilon}=0.6$ is lower than the stress amplitude at $\mathrm{R}_{\epsilon}=0$ (see Fig. 3c). If the crack growth rate is plotted against the stress intensity factor range, $\Delta K \propto \Delta \sigma$, it can be observed that the growth rate is faster at higher $\mathrm{R}_{\epsilon}$ for the same crack growth driving force [25]. This behaviour is consistent with plasticity induced crack closure arguments.

In the high strain experiments strain controlled loading is used. In order to achieve similar conditions in the FE-analyses a displacement control algorithm was applied in Abaqus. In this algorithm the prescribed cyclic displacements of the top nodes of the FE-models are controlled by a user subroutine DISP. Moreover, the displacements at the node corresponding to the location of the extensometer, i.e. at the middle of the short side of the specimen cross-section $6 \mathrm{~mm}$ from the crack plane, are checked after each time increment by the user subroutine URDFIL, cf. [20]. As a result, the measured strain at this virtual extensometer position is controlling the loading.

For the FE-analyses, two of the constitutive models presented in Section 3.1 are used. In Section 3.1 it was shown that all three evaluated material models could represent the experiments with good accuracy. However, the results from the Chaboche with threshold model and the Ohno-Wang model were similar. Therefore, only the Ohno-Wang model is implemented in a UMAT-routine [20] and used for the FE-analyses. Furthermore, the evaluation of the material models in Section 3.1 also showed that the Chaboche model had a drawback of an overly stiff kinematic hardening effect, which resulted in the need for 
two separate parameter sets for the two loading conditions, $R_{\epsilon}=0$ and $R_{\epsilon}=0.6$. Although this implies a limitation of the model for simulating general cases of loading, the Chaboche model's accessibility in commercial software and computational efficiency still makes it an interesting candidate and it is therefore used in the FE-analyses.

\subsection{Predicted load drop}

Nominal stresses are extracted from FE-analyses at the cycle corresponding to the three different crack sizes analysed (see Table 5). For the Chaboche model analyses have been conducted for $\mathrm{R}_{\epsilon}=0$ and $\mathrm{R}_{\epsilon}=0.6$ and for the three different sets of cycle-scaled material parameters. These results are plotted in Fig. $7 \mathrm{a}$ in the form of relative reduction in maximum stress as a function of the relative reduction in cross-section area. The relative reduction in maximum stress is normalised by the semi-stable stress value, reached after 500 cycles, for each simulation. In Fig. 7a experimental results of relative reduction in maximum stress versus relative reduction in cross-section area are also given for all high strain tests. It can be noted that most of the FE-results underestimate the load drop for the largest crack of 2 $\mathrm{mm}$. For this crack length a relative reduction in maximum stress of $4.7-9.5 \%$ for $\mathrm{R}_{\epsilon}=0$ and of $6.1-7.1 \%$ for $R_{\epsilon}=0.6$, is obtained. These results can be compared to experiments, for which a relative reduction in maximum stress of $9.5 \%$ is observed for a $2 \mathrm{~mm}$ crack. Moreover, the load drops obtained in the FE-analyses, if compared to experiments, would correspond to crack lengths of 1.7-2.0 mm and 1.8-1.9 mm, for $R_{\epsilon}=0$ and $R_{\epsilon}=0.6$ respectively. It can be observed that the number of cycles needed to propagate the crack to a size of $2 \mathrm{~mm}$ (1400 cycles) is outside the range for which the material parameters have been calibrated. Although the model response is similar within the calibrated interval of cycles, as observed in Section 3.1, the extrapolated model response may be causing a scatter in the result for the larger cracks.

In Fig. 7b FE-results of the relationship between load drop and relative reduction in crosssectional area are shown for the Ohno-Wang model. Only results for cycle-scales of 1:10 and 1:100 are given, due to the computationally demanding analyses. It can be observed that the results for the analyses using the Ohno-Wang model correspond as well to experiments as the results from the analyses using the Chaboche model. For the $2 \mathrm{~mm}$ crack a relative reduction in maximum stress of $6.7-9.9 \%$ for $R_{\epsilon}=0$ and of $5.3-6.7 \%$ for $R_{\epsilon}=0.6$, is obtained. Compared to experiments these load drops would correspond to crack lengths of 1.8-2.0 mm and 1.75$1.85 \mathrm{~mm}$, respectively. In conclusion, the Ohno-Wang model also results in a scatter for the analyses of the larger cracks. However, the advantage of the Ohno-Wang model, compared to the Chaboche model, is that it can be used for loading conditions not used in the calibration procedure, as discussed in Section 3.1.

From Fig. 7 it can be concluded that the simulations using the cycle-scaled parameters do not significantly affect the accuracy of the results. This can be observed both for the Chaboche model and the Ohno-Wang model. It can also be mentioned that reduction in computation time, for the cycle-scaling scheme, is in the order of the reduction in the required load cycles. Therefore, resulting in 10 or 100 times faster simulations.

\subsection{Influence of extensometer position}

In LCF testing the position of the extensometer relative to the crack is of importance for the measured load drop. To show this influence, four virtual extensometer locations were 


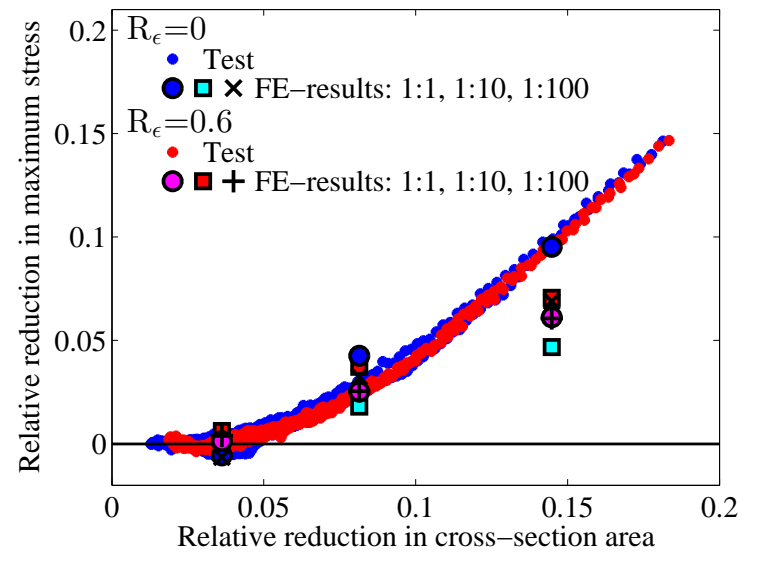

(a)

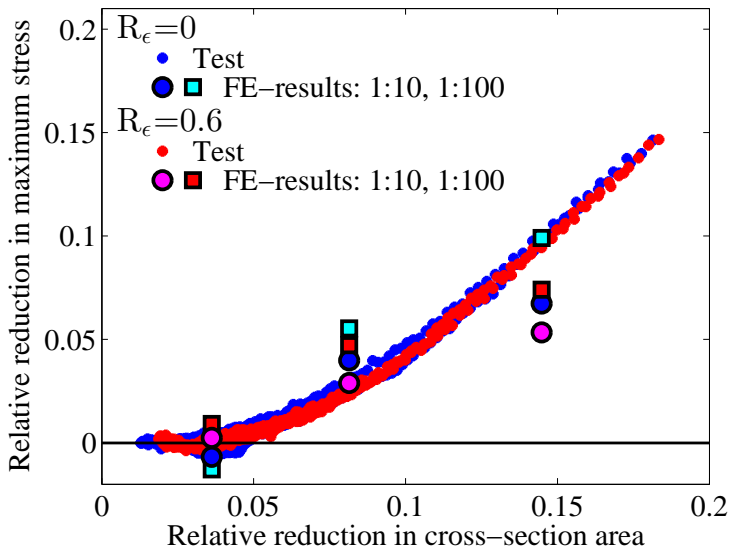

(b)

Figure 7: Relationship between relative reduction in load carrying area and relative reduction in maximum stress for all tested high strain specimens. Also included are FE-results from analyses with crack lengths 1, 1.5 and $2 \mathrm{~mm}$ using cycled-scaled material parameters for (a) the Chaboche model and (b) the Ohno-Wang model.

evaluated for the FE-models of the high strain crack growth specimen. The four locations are shown in Fig. 8, for the FE-model of a specimen with a $2 \mathrm{~mm}$ crack, and can be described as:

- Extensometer location 1 (reference case): Original position used in the experiment setup, in the middle of the short side of the specimen cross-section, $6 \mathrm{~mm}$ from the crack plane

- Extensometer location 2: Centred above the crack, in the middle of the long side of the specimen cross-section, $6 \mathrm{~mm}$ from the crack plane

- Extensometer location 3: Centred behind the crack, in the middle of the long side of the specimen cross-section, $6 \mathrm{~mm}$ from the crack plane

- Extensometer location 4: At the middle of the short side of the specimen cross-section, $15 \mathrm{~mm}$ from the crack plane

All virtual extensometers measure the strain from the symmetry crack plane, except for the last one, extensometer location 4. For this location, the strain is measured between extensometer 1 and 4, giving a situation similar to that of a crack occurring outside the extensometer gauge length in an LCF test.

FE-results for the load drop when using the different virtual extensometer locations are given in Fig. 9. For these simulations the Chaboche model with parameters calibrated for cycle-scale 1:1 were used. It can be noted that the influence of the extensometer position clearly increases with the crack size. In Fig. 9a results are given for the loading condition of $\mathrm{R}_{\epsilon}=0$. It can be observed that the result of measuring the strain behind the crack (extensometer location 3) or outside the crack (extensometer location 4) gives a lower load drop 


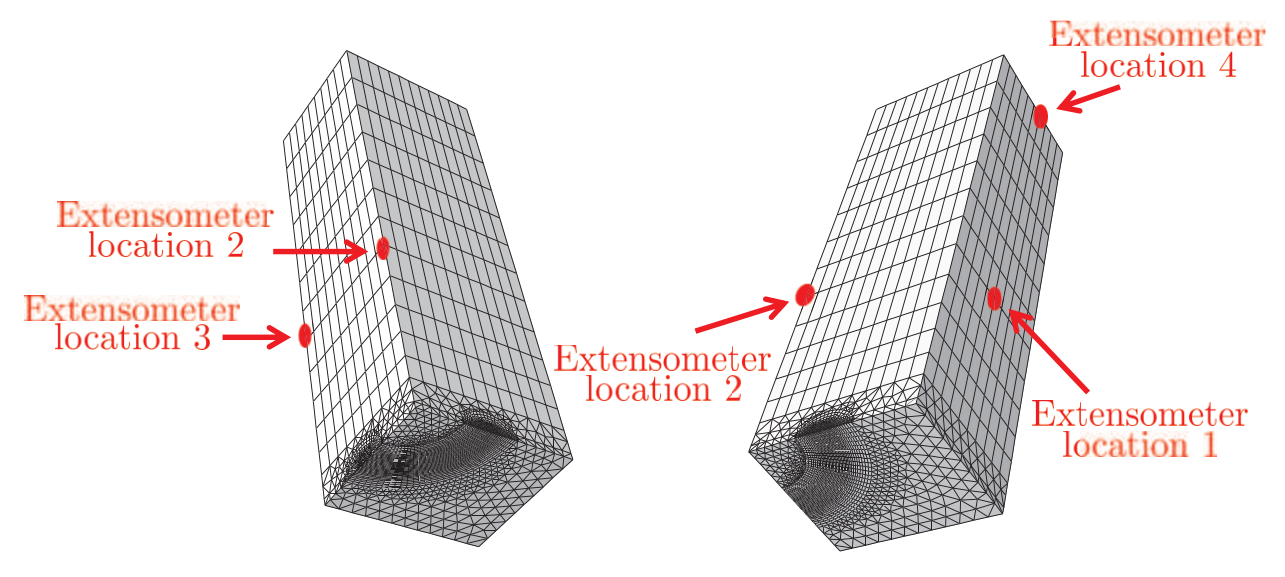

Figure 8: FE-model of the test specimen with a crack length of $2 \mathrm{~mm}$ indicating the four locations for the virtual extensometers.

than the reference position (extensometer location 1). This would imply that for interrupted testing based on the X \% load drop criterion a larger crack would be present compared to the case where extensometer location 1 is used. The largest difference in load drop compared to the original extensometer location is obtained when measuring the strain from a position centred above the crack (extensometer location 2). This result gives a much higher relative reduction in maximum stress, $17 \%$ compared to $9.5 \%$ for the original position. In Fig. $9 \mathrm{~b}$ results are given for the loading condition of $R_{\epsilon}=0.6$. From this figure it can be noted that the results follow the trend observed for analyses with $\mathrm{R}_{\epsilon}=0$.

The results in Fig. 9 show a scatter in load drop of $5-17 \%$ for $R_{\epsilon}=0$ and $1-11 \%$ for $R_{\epsilon}=0.6$, for a $2 \mathrm{~mm}$ crack. This scatter due to the extensometer location is much larger than the scatter observed for the different material models and the cycle-scaled parameters presented in Section 4.2. Furthermore, the scatter in load drop due to the extensometer position would be even more pronounced for round bar specimens, as the compliance will be more affected by the crack, which approach a through-crack as it grows. Additionally, it can be pointed out that simulations like these can be used to estimate the scatter in LCF life due to position of crack initiation sites.

\section{Conclusions}

- To simulate the relationship between reductions of area and drop in maximum load during high strain fatigue crack growth testing it is vital to capture both maximum/minimum stresses and mean stress relaxation in the constitutive model. In this work, three constitutive models, the Chaboche model, the Ohno-Wang model and the Chaboche with threshold model, were evaluated. It was shown that all three models could be used to model the mean stress relaxation. However, the overly stiff kinematic hardening effect necessary for the Chaboche model requires that material parameters are cali- 


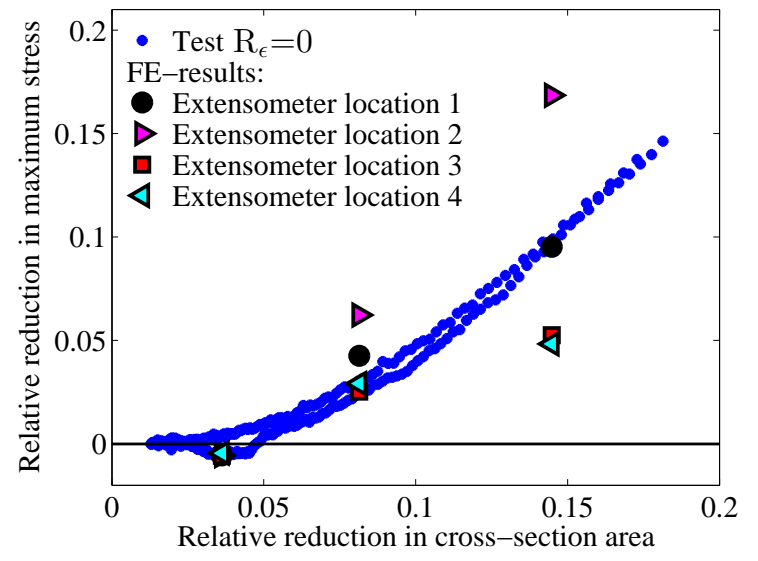

(a)

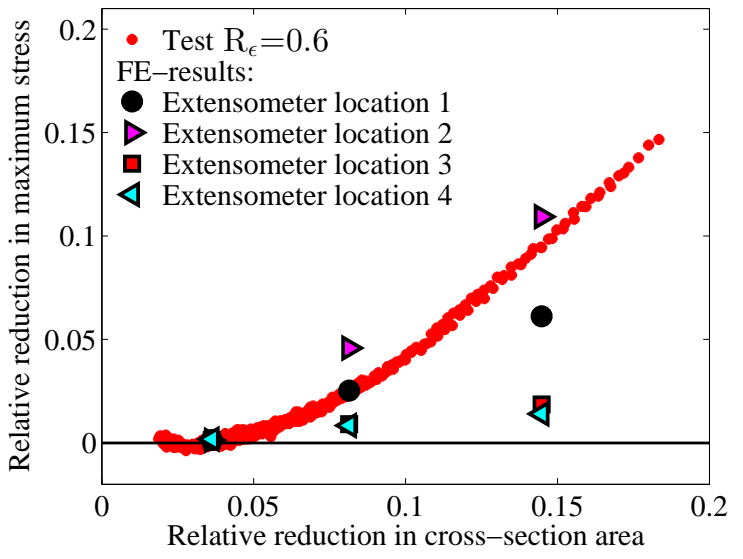

(b)

Figure 9: Relationship between relative reduction in load carrying area and relative reduction in maximum stress for the tested high strain specimens and FE-results for analyses with four different locations for the virtual extensometer and loading conditions of (a) $R_{\epsilon}=0$ and (b) $\mathrm{R}_{\epsilon}=0.6$.

brated for the applied loading conditions. Therefore, the Ohno-Wang and Chaboche with threshold models can be considered as better choices for cases of general loading.

- Accounting for mean stress relaxation in simulations is computational expensive, as the simulations must be run for the same number of cycles as in the tests. Therefore, an approach of cycle-scaling of experiments and material parameters were proposed and implemented. It was shown that only the material parameters controlling the mean stress relaxation were needed to be re-calibrated for the cycle-scaled experiments. Furthermore, the cycle-scaling did not significantly affect the accuracy of the model predictions and the computation time is linearly with the cycle-scaling.

- From the FE-simulations of the relative reduction in maximum stress for three different crack lengths, it could be concluded that a load drop - crack length relationship could be established using the Chaboche or Ohno-Wang models. Although a difference as compared to the experimental results was obtained for the largest crack of $2 \mathrm{~mm}$, the results are promising for predicting the load drop - crack length relationship for arbitrary specimen and crack geometries. For the Ohno-Wang model predictions of this relationship could also be obtained for more general loadings. In the simulations a constant crack length has been assumed. It is suggested in future work to include crack propagation and to study how this will influence the results.

- FE-results with a virtual extensometer showed that the extensometer position relative to the crack is highly important for predicting an accurate load drop-crack length relationship.

- In conclusion, the proposed methodology could be used to estimate the crack length from load-drop in LCF testing. Crack lengths could also be calculated from previously 
performed testing, provided that the crack initiation sites have been registered. Finally, the results may also be used to obtain an estimate of the scatter in LCF load drop due to different crack initiation sites for the test specimens.

\section{Acknowledgements}

This work has been founded by the Swedish National Aeronautical Research Program (NFFP) which is gratefully acknowledged.

\section{References}

[1] K. Bhanu Sankara Rao, M. Valsan, R. Sandhya, S. K. Ray, S. L. Mannan, P. Rodriquez, On the failure condition in strain-controlled low-cycle fatigue, International Journal of Fatigue 7 (1985) 141-147.

[2] A. E-606, Standard test method for strain-controlled fatigue testing, in: Annual Book of ASTM Standards, ASTM, West Conshohocken, 2012.

[3] G. Thomas, R. Hales, J. Ramsdale, R. Suhr, G. Sumner, A code of practice for constantamplitude low cycle fatigue testing at elevated temperatures, Fatigue \& Fracture of Engineering Materials and Structures 12 (1989) 135-153.

[4] R. Skelton, Cyclic hardening, softening, and crack growth during high temperature fatigue, Material Science and Technology 9 (1993) 1001-1008.

[5] M. Kitagawa, K. Yamaguchi, Y. Fukuda, R. Komine, H. Hirata, Analysis on VAMAS low cycle fatigue Round Robin test in Japan, ISIJ International 33 (1993) 817-824.

[6] R. Brommesson, M. Hörnqvist, M. Ekh, Low-cycle fatigue crack growth in Ti-6242 at elevated temperature, Advanced Materials Research 891-892 (2014) 422-427.

[7] S. Bari, T. Hassan, Anatomy of coupled constitutive models for ratchetting simulation, International Journal of Plasticity 16 (2000) 381 - 409.

[8] J. Chaboche, A review of some plasticity and viscoplasticity constitutive theories, International Journal of Plasticity 24 (2008) 1642 - 1693.

[9] G. Johansson, M. Ekh, On the modeling of large ratcheting strains with large time increments, Engineering Computations 24 (2007) 221 - 236.

[10] R. Brommesson, M. Ekh, Modelling of cyclic behaviour of Haynes 282 at elevated temperatures, Materials at High Temperatures 31 (2014) 121-130.

[11] R. Peerlings, W. Brekelmans, R. Borst, M. Geers, Gradient-enhanced damage modelling of high-cycle fatigue, International Journal for Numerical Methods in Engineering 49 (2000) 1547-1569.

[12] A. Suiker, R. Borst, A numerical model for the cyclic deterioration of railway tracks, International Journal for Numerical Methods in Engineering 57 (2003) 441-470. 
[13] P. Armstrong, C. Frederick, A mathematical representation of the multiaxial Bauschinger effect, Report RD/B/N/731, Central Electricity Geneating Board, Berkely UK, (1966).

[14] J. Chaboche, Constitutive equations for cyclic plasticity and cyclic viscoplasticity, International Journal of Plasticity 5 (1989) $247-302$.

[15] N. Ohno, J. Wang, Kinematic hardening rules with critical state of dynamic recovery, Part I: Formulation and basic features for ratchetting behaviour, International Journal of Plasticity 9 (1993) 375-390.

[16] J. Chaboche, On some modifications of kinematic hardening to improve the description of ratchetting effects, International Journal of Plasticity 7 (1991) 661-678.

[17] J. Chaboche, Modelling of ratchetting: evaluation of various approaches, European Journal of Mechanics - A/Solids 13 (1994) 501-518.

[18] J. Chaboche, P. Kanouté, F. Azzouz, Cyclic inelastic constitutive equations and their impact on the fatigue life predicitions, International Journal of Plasticity 35 (2012) 44 -66 .

[19] A. Coles, R. Johnson, H. Popp, Utility of surface-flawed tensile bars in cyclic life studies, ASME Journal of Engineering Matererials and Technology 98 (1976) 305-315.

[20] ABAQUS 6.13, Abaqus Documentation, Dassault Systems Simulia Corp., Providence, RI, USA, 2013.

[21] M. Becker, H. Hackenberg, A constitutive model for rate dependent and rate independent inelasticity. Application to IN718, International Journal of Plasticity 27 (2010) 596 - 619.

[22] D. Gustafsson, J. Moverare, K. Simonsson, S. Sjöström, Modeling of the constitutive behavior of Inconel 718 at intermidiate temperatures, Journal of Engineering for Gas Turbines and Power 133 (2011) 1-4.

[23] R. Brommesson, M. Ekh, Experiments and modelling of the cyclic behaviour of Haynes 282, Technische Mechanik 32 (2012) 130 - 145.

[24] R. Hales, S. Holdsworth, M. Donnell, I. Perrin, R. Skelton, A code of practice for the determination of cyclic stress-strain data, Materials at High Temperatures 19 (2002) $165-185$.

[25] M. Hörnqvist, T. Månsson, Fatigue crack growth in Ti-6242 under elasto-plastic loading conditions, Procedia Engineering 2 (2010) 223-229. 